This article was downloaded by: [North Carolina State University]

On: 26 May 2009

Access details: Access Details: [subscription number 783677828]

Publisher Taylor \& Francis

Informa Ltd Registered in England and Wales Registered Number: 1072954 Registered office: Mortimer House, 37-41 Mortimer Street, London W1T 3JH, UK

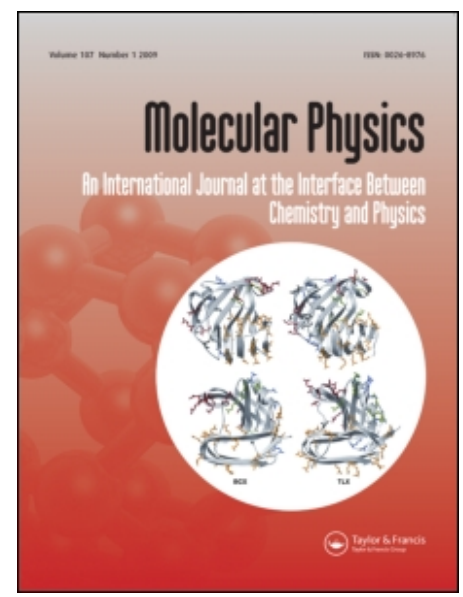

Molecular Physics

Publication details, including instructions for authors and subscription information:

http://www.informaworld.com/smpp/title content=t713395160

\title{
An efficient procedure for the study of inhomogeneous liquids
}

F. Lado ${ }^{a}$

${ }^{a}$ Department of Physics, North Carolina State University, Raleigh, NC 27695-8202, USA

First Published:February2009

To cite this Article Lado, F.(2009)'An efficient procedure for the study of inhomogeneous liquids',Molecular Physics,107:4,301 — 308

To link to this Article: DOI: $10.1080 / 00268970802603531$

URL: http://dx.doi.org/10.1080/00268970802603531

\section{PLEASE SCROLL DOWN FOR ARTICLE}

\footnotetext{
Full terms and conditions of use: http://www.informaworld.com/terms-and-conditions-of-access.pdf

This article may be used for research, teaching and private study purposes. Any substantial or systematic reproduction, re-distribution, re-selling, loan or sub-licensing, systematic supply or distribution in any form to anyone is expressly forbidden.

The publisher does not give any warranty express or implied or make any representation that the contents will be complete or accurate or up to date. The accuracy of any instructions, formulae and drug doses should be independently verified with primary sources. The publisher shall not be liable for any loss, actions, claims, proceedings, demand or costs or damages whatsoever or howsoever caused arising directly or indirectly in connection with or arising out of the use of this material.
} 


\title{
INVITED ARTICLE
}

\section{An efficient procedure for the study of inhomogeneous liquids}

\author{
F. Lado* \\ Department of Physics, North Carolina State University, Raleigh, NC 27695-8202, USA
}

(Received 20 October 2008; final version received 31 October 2008)

\begin{abstract}
A complete description of an inhomogeneous fluid requires knowing not only its density $\rho(\boldsymbol{r})$ at any point $\boldsymbol{r}$ in space, but also its pair distribution function $g\left(\boldsymbol{r}_{1}, \boldsymbol{r}_{2}\right)$. These can be found from coupled integral equations, but even in cases with homogeneity in two of the three dimensions, such solutions in direct space are cumbersome enough to have earned the task a reputation as 'computationally intensive'. Here we propose using purpose-built basis functions that are orthogonal with weight function $\rho(\boldsymbol{r})$ to expedite the work. We find that these not only produce a satisfying simplification of the formalism, but also reduce the computing effort needed to something on the order of $1 \%$ of that of the earlier calculations in direct space.
\end{abstract}

Keywords: inhomogeneous liquids; integral equations; Ornstein-Zernike equation; special orthogonal polynomials

\section{Introduction}

A complete description of an inhomogeneous fluid requires knowing not only its density $\rho(\boldsymbol{r})$ at any point $\boldsymbol{r}$ in space, but also its pair distribution function $g\left(\boldsymbol{r}_{1}, \boldsymbol{r}_{2}\right)$ describing the spatial correlation of two fluid particles at locations $\boldsymbol{r}_{1}$ and $\boldsymbol{r}_{2}$. By the definition of inhomogeneous, this latter quantity is no longer just a function of $r_{12}=\left|\boldsymbol{r}_{1}-\boldsymbol{r}_{2}\right|$ and therein lies the special challenge of solving Ornstein-Zernike (OZ) type integral equations for such systems. In many cases, the symmetry of a specific system will allow the general inhomogeneous OZ equation [1],

$$
h\left(\boldsymbol{r}_{1}, \boldsymbol{r}_{2}\right)=c\left(\boldsymbol{r}_{1}, \boldsymbol{r}_{2}\right)+\int \mathrm{d} \boldsymbol{r}_{3} h\left(\boldsymbol{r}_{1}, \boldsymbol{r}_{3}\right) \rho\left(\boldsymbol{r}_{3}\right) c\left(\boldsymbol{r}_{3}, \boldsymbol{r}_{2}\right),
$$

to be partially deconvoluted using an integral transform, leaving a final one-dimensional integral unevaluated. (Here $h\left(\boldsymbol{r}_{1}, \boldsymbol{r}_{2}\right)=g\left(\boldsymbol{r}_{1}, \boldsymbol{r}_{2}\right)-1$ is the inhomogeneous pair correlation function and $c\left(\boldsymbol{r}_{1}, \boldsymbol{r}_{2}\right)$ the inhomogeneous direct correlation function effectively defined by the $\mathrm{OZ}$ equation.) Written in discrete form on a finite grid with summation replacing integration, this final equation for the transforms can then be interpreted as a matrix problem in direct space and solved accordingly. This usually entails a large number of spatial grid points and so the inhomogeneous liquid problem has acquired a reputation as computationally intensive (by integral equation standards, leaving aside simulation). In this note, for the Festschrift on the occasion of Jean-Jacques Weis' retirement, we describe how the remaining integral can also be analytically evaluated and the $\mathrm{OZ}$ equation reduced to an algebraic relation among expansion coefficients alone, just as for homogeneous molecular liquids, with an attendant sharp reduction in computing effort. This simplification is accomplished by the construction of tailored functions designed to be orthogonal with weight function $\rho(\boldsymbol{r})$.

The inhomogeneous OZ equation, coupled with an exact relation between the one-body and two-body functions (described below), was first solved for fluids in contact with a single hard wall by Sokołowski $[2,3]$ and by Plischke and Henderson [4,5]. Liquids between two parallel walls (a planar slit) have been extensively and elegantly studied by Kjellander and Sarman [6-9]. These works all use a Hankel transform to eliminate two of the three integrals in the inhomogeneous $\mathrm{OZ}$ equation. Attard [10] has shown how the two angular integrals can be eliminated using a Legendre transformation for fluids with spherical inhomogeneity. A clever application is then created by letting the spherical inhomogeneity arise from a fluid particle fixed at the origin of coordinates, so that an $n$-body distribution function in the inhomogeneous liquid becomes an $(n+1)$-body distribution function in the original homogeneous system. Familiar approximations now made at the nominal two-body level produce far better results [11-14] than when made at the conventional two-body level in a homogeneous formulation.

\footnotetext{
*Email: fred_lado@ncsu.edu 
In the next section, we describe a procedure that eliminates the surviving one-dimensional integral that complicates the task in the works cited above and finally converts the $\mathrm{OZ}$ equation into a purely algebraic expression for the expansion coefficients alone. For specificity, we use the language of a planar slit; revising the notation for other geometries is straightforward. Applications of the procedure are left to later works.

\section{Reducing the $\mathrm{OZ}$ equation to algebra}

A planar slit of width $L$ is allowed to have indefinite wall extent so no boundary conditions are needed in the plane of the walls. The inhomogeneity then occurs perpendicular to the wall planes, which we denote conventionally as the $z$ direction. The density is now a one-dimensional function, $\rho(z)$, and pair functions such as $h\left(\boldsymbol{r}_{1}, \boldsymbol{r}_{2}\right)$ can be described using coordinates $R_{12} \equiv\left[\left(x_{1}-x_{2}\right)^{2}+\left(y_{1}-y_{2}\right)^{2}\right]^{1 / 2}, \quad z_{1}, \quad$ and $z_{2}$ : thus, $h\left(\boldsymbol{r}_{1}, \boldsymbol{r}_{2}\right)=h\left(R_{12}, z_{1}, z_{2}\right)$, etc. The $\mathrm{OZ}$ equation now reads

$$
\begin{aligned}
h\left(R_{12}, z_{1}, z_{2}\right)= & c\left(R_{12}, z_{1}, z_{2}\right)+\int_{-L / 2}^{L / 2} \mathrm{~d} z_{3} \rho\left(z_{3}\right) \\
& \times \int \mathrm{d} \boldsymbol{R}_{3} h\left(R_{13}, z_{1}, z_{3}\right) c\left(R_{32}, z_{3}, z_{2}\right) .
\end{aligned}
$$

The first step in simplifying this equation is to use the homogeneity of the pair functions in the $\boldsymbol{R}$ plane to deconvolute it with a Hankel transform [2-9]:

$$
\begin{aligned}
\hat{h}\left(K, z_{1}, z_{2}\right) & =\int \mathrm{d} \boldsymbol{R} h\left(R, z_{1}, z_{2}\right) \mathrm{e}^{-i \boldsymbol{K} \cdot \boldsymbol{R}} \\
& =2 \pi \int_{0}^{\infty} \mathrm{d} R R h\left(R, z_{1}, z_{2}\right) J_{0}(K R), \\
h\left(R, z_{1}, z_{2}\right) & =\frac{1}{(2 \pi)^{2}} \int \mathrm{d} \boldsymbol{K} \hat{h}\left(K, z_{1}, z_{2}\right) \mathrm{e}^{i \boldsymbol{K} \cdot \boldsymbol{R}} \\
& =\frac{1}{2 \pi} \int_{0}^{\infty} \mathrm{d} K K \hat{h}\left(K, z_{1}, z_{2}\right) J_{0}(K R),
\end{aligned}
$$

where $J_{0}(x)$ is the Bessel function of order zero. We then obtain

$$
\begin{aligned}
\hat{h}\left(K, z_{1}, z_{2}\right)= & \hat{c}\left(K, z_{1}, z_{2}\right) \\
& +\int_{-L / 2}^{L / 2} \mathrm{~d} z_{3} \rho\left(z_{3}\right) \hat{h}\left(K, z_{1}, z_{3}\right) \hat{c}\left(K, z_{3}, z_{2}\right),
\end{aligned}
$$

with one remaining integral. This is the version of the $\mathrm{OZ}$ equation that all earlier papers have used. In practice, of course, the equation must be solved on a finite grid of points on the $z$ axis. If we use $N_{z}$ points, their spacing is $\Delta z=L /\left(N_{z}-1\right)$ and the discrete version is

$$
\hat{h}_{i j}(K)=\hat{c}_{i j}(K)+\sum_{k=-N_{z} / 2}^{N_{z} / 2} \Delta z \rho_{k} \hat{h}_{i k}(K) \hat{c}_{k j}(K),
$$

where $\rho_{j}=\rho\left(z_{j}\right), \hat{h_{i j}}(K)=\hat{h}\left(K, z_{i}, z_{j}\right)$, etc., with $z_{j}=j \Delta z$. A more symmetric version of the equation can be obtained by multiplying through with $\left(\rho_{i} \rho_{j}\right)^{1 / 2}$. In any case, one now has a matrix equation that can be manipulated using standard matrix techniques. Since a large number of grid points is generally needed for an accurate numerical representation, the result is a calculation that is often deemed to be 'computationally intensive'.

The usual remedy for this sort of problem is an expansion of the $z$ dependence in orthogonal functions, with the critical expectation that far fewer expansion coefficients will be needed than grid points on the $z$ axis. Here that would ordinarily mean expansions in plane waves (using periodic boundary conditions),

$$
\begin{aligned}
E_{m}(z) & \equiv \mathrm{e}^{i m(2 \pi z / L)} \\
& =T_{m}[\cos (2 \pi z / L)]+i V_{m}[\cos (2 \pi z / L)],
\end{aligned}
$$

where $m$ is a positive or negative integer. (For brevity, we will put

$$
\xi \equiv \cos (2 \pi z / L)
$$

in the remainder of this work.) We recognize here $T_{m}(\xi)=\cos [m(2 \pi z / L)]$ as the type I Chebyshev polynomial of order $m$ and $V_{m}(\xi)=\sin [m(2 \pi z / L)]$ the associated Chebyshev function (not a polynomial) of order $m$ [15]. The familiar exponential functions $E_{m}(z)$ of course have the orthonormalization

$$
\frac{1}{L} \int_{-L / 2}^{L / 2} \mathrm{~d} z E_{m}(z) E_{m^{\prime}}^{*}(z)=\delta_{m m^{\prime}},
$$

where the asterisk denotes complex conjugate, for $m$, $\mathrm{m}^{\prime}$ positive or negative integers. But if we now put into Equation (5)

$$
\hat{h}\left(K, z_{1}, z_{3}\right)=\sum_{m_{1}, m_{3}=-\infty}^{\infty} \hat{H}_{m_{1} m_{3}}(K) E_{m_{1}}\left(z_{1}\right) E_{m_{3}}^{*}\left(z_{3}\right),
$$

and similarly for $\hat{c}\left(K, z_{3}, z_{2}\right)$, the integral that turns up is not Equation (9), but rather

$$
\frac{1}{L} \int_{-L / 2}^{L / 2} \mathrm{~d} z \rho(z) E_{m}(z) E_{m^{\prime}}^{*}(z),
$$

and the invaluable property of orthogonality is lost.

The essential proposal of this work is that one should simply replace the familiar $E_{m}(z)$ with specially 
constructed basis functions $\mathcal{E}_{m}(z)$ that are specifically designed to be orthogonal with weight function $\rho(z)$. Preferring a dimensionless weight function, we define the mean density

$$
\bar{\rho}=\frac{1}{L} \int_{-L / 2}^{L / 2} \mathrm{~d} z \rho(z),
$$

and put $\rho(z)=\bar{\rho} f(z)$. Then the tailored orthogonal functions will be constructed to satisfy

$$
\frac{1}{L} \int_{-L / 2}^{L / 2} \mathrm{~d} z f(z) \mathcal{E}_{m}(z) \mathcal{E}_{m^{\prime}}^{*}(z)=\delta_{m m^{\prime}}
$$

again for $m, m^{\prime}$ positive or negative integers, while $f(z)$ itself is normalized to unity,

$$
\frac{1}{L} \int_{-L / 2}^{L / 2} \mathrm{~d} z f(z)=1
$$

Now we may put

$$
\hat{h}\left(K, z_{1}, z_{3}\right)=\sum_{m_{1}, m_{3}=-\infty}^{\infty} \hat{h}_{m_{1} m_{3}}(K) \mathcal{E}_{m_{1}}\left(z_{1}\right) \mathcal{E}_{m_{3}}^{*}\left(z_{3}\right)
$$

and similarly for $\hat{c}\left(K, z_{3}, z_{2}\right)$, so that the $\mathrm{OZ}$ equation finally becomes a relation among expansion coefficients alone,

$$
\hat{h}_{m_{1} m_{2}}(K)=\hat{c}_{m_{1} m_{2}}(K)+\bar{\rho} L \sum_{m_{3}=-\infty}^{\infty} \hat{h}_{m_{1} m_{3}}(K) \hat{c}_{m_{3} m_{2}}(K) .
$$

We note that coefficients of all pair functions satisfy the symmetries

$$
\hat{c}_{m_{1} m_{2}}(K)=\hat{c}_{m_{2} m_{1}}(K)=\hat{c}_{\bar{m}_{2} \bar{m}_{1}}(K)=\hat{c}_{\bar{m}_{1} \bar{m}_{2}}(K),
$$

where $\bar{m} \equiv-m$. As usual, it is numerically preferable to eliminate $h$ from the $\mathrm{OZ}$ equation and solve instead for the continuous 'series' function [16] $s=h-c$, for which we obtain

$$
\hat{s}_{m_{1} m_{2}}(K)=\bar{\rho} L \sum_{m_{3}=-\infty}^{\infty}\left[\hat{s}_{m_{1} m_{3}}(K)+\hat{c}_{m_{1} m_{3}}(K)\right] \hat{c}_{m_{3} m_{2}}(K) .
$$

This is finally solved for the $\hat{s}_{m_{1} m_{2}}(K)$; in matrix notation,

$$
\hat{\mathbf{S}}(K)=\bar{\rho} L \hat{\mathbf{C}}(K) \hat{\mathbf{C}}(K)[\mathbf{I}-\bar{\rho} L \hat{\mathbf{C}}(K)]^{-1},
$$

where $\hat{\mathbf{S}}(K)$ and $\hat{\mathbf{C}}(K)$ are square matrices with elements $\hat{s}_{m_{1} m_{2}}(K)$ and $\hat{c}_{m_{1} m_{2}}(K)$, respectively, and $\mathbf{I}$ is the unit matrix. (Given the symmetries (16), only one-quarter of the elements of $\hat{\mathbf{C}}(K)$, etc., are unique.) The notable feature of Equation (18) is that it is identical to that of a homogeneous system. All of the inhomogeneity is built into the basis functions.

The construction of the tailored basis functions $\mathcal{E}_{m}(z)$,

$$
\begin{gathered}
\mathcal{E}_{m}(z)=\mathcal{T}_{m}(\xi)+i \mathcal{V}_{m}(\xi), \\
\mathcal{E}_{-m}(z)=\mathcal{E}_{m}^{*}(z),
\end{gathered}
$$

for $m \geq 0$, where $\mathcal{T}_{m}(\xi)$ and $\mathcal{V}_{m}(\xi)$ are the needed generalized Chebyshev functions, is outlined in Section 4. We turn first to the relationship between $\rho(z)$ and the pair functions.

\section{Calculating the density profile}

There are three exact equations that connect the onebody and two-body functions. Triezenberg and Zwanzig [17], Lovett et al. [18], and Wertheim [19] have shown that

$$
\begin{aligned}
\frac{\mathrm{d} \ln \rho\left(z_{1}\right)}{\mathrm{d} z_{1}}= & -\frac{\mathrm{d} \beta v_{\text {ext }}\left(z_{1}\right)}{\mathrm{d} z_{1}}-\int_{-L / 2}^{L / 2} \mathrm{~d} z_{2} \rho\left(z_{2}\right) \\
& \times \int \mathrm{d} \boldsymbol{R}_{2} h\left(R_{12}, z_{1}, z_{2}\right) \frac{\mathrm{d} \beta v_{\text {ext }}\left(z_{2}\right)}{\mathrm{d} z_{2}} \\
= & -\frac{\mathrm{d} \beta v_{\text {ext }}\left(z_{2}\right)}{\mathrm{d} z_{1}}-\int_{-L / 2}^{L / 2} \mathrm{~d} z_{2} \rho\left(z_{2}\right) \hat{h}\left(0, z_{1}, z_{2}\right) \\
& \times \frac{\mathrm{d} \beta v_{\text {ext }}\left(z_{2}\right)}{\mathrm{d} z_{2}}
\end{aligned}
$$

where $v_{\text {ext }}(z)$ is the external potential (here the walls of the planar slit) and $\beta=\left(k_{B} T\right)^{-1}$ the inverse Kelvin temperature, with $k_{B}$ Boltzmann's constant. Alternatively, passing through the $\mathrm{OZ}$ equation, this can be written as

$$
\begin{aligned}
\frac{\mathrm{d} \ln \rho\left(z_{1}\right)}{\mathrm{d} z_{1}}= & -\frac{\mathrm{d} \beta v_{\text {ext }}\left(z_{1}\right)}{\mathrm{d} z_{1}}+\int_{-L / 2}^{L / 2} \mathrm{~d} z_{2} \\
& \times \int \mathrm{d} \boldsymbol{R}_{2} c\left(R_{12}, z_{1}, z_{2}\right) \frac{\mathrm{d} \rho\left(z_{2}\right)}{\mathrm{d} z_{2}} \\
= & -\frac{\mathrm{d} \beta v_{\text {ext }}\left(z_{1}\right)}{\mathrm{d} z_{1}}+\int_{-L / 2}^{L / 2} \mathrm{~d} z_{2} \rho\left(z_{2}\right) \hat{c}\left(0, z_{1}, z_{2}\right) \\
& \times \frac{\mathrm{d} \ln \rho\left(z_{2}\right)}{\mathrm{d} z_{2}} .
\end{aligned}
$$

The third exact equation is the first member of the Yvon-Born-Green hierarchy [20], which, for the sake of brevity, we will not examine here.

Because $f(z)$ is highly structured or even discontinuous in the vicinity of the walls, we will solve for the 
smoother and continuous combination $f(z)$ $\exp \left[\beta v_{\text {ext }}(z)\right]$. We work first with Equation (21) and put

$$
\begin{gathered}
\ln \left[f(z) \mathrm{e}^{\beta v_{\text {ext }}(z)}\right]=\sum_{m=0}^{\infty} A_{m} \mathcal{T}_{m}(\xi), \\
\hat{h}\left(0, z_{1}, z_{2}\right)=\sum_{m_{1}, m_{2}=-\infty}^{\infty} \hat{h}_{m_{1} m_{2}}(0) \mathcal{E}_{m_{1}}\left(z_{1}\right) \mathcal{E}_{m_{2}}^{*}\left(z_{2}\right),
\end{gathered}
$$

where we now seek to calculate the coefficients $A_{m}$. (Note that $A_{0}$ is determined by normalization.) We have for Equation (21),

$$
\begin{aligned}
\sum_{m=1}^{\infty} A_{m} \frac{\mathrm{d} \mathcal{T}_{m}(\xi)}{\mathrm{d} z}= & -\sum_{m_{1}, m_{2}=-\infty}^{\infty} \bar{\rho} \hat{h}_{m_{1} m_{2}}(0) \mathcal{E}_{m_{1}}(z) \\
& \times \int_{-L / 2}^{L / 2} \mathrm{~d} z^{\prime} f\left(z^{\prime}\right) \mathcal{E}_{m_{2}}^{*}\left(z^{\prime}\right) \frac{\mathrm{d} \beta v_{\text {ext }}\left(z^{\prime}\right)}{\mathrm{d} z^{\prime}} .
\end{aligned}
$$

Now multiply this equation by $(i / L) f(z) \mathcal{E}_{m^{\prime}}^{*}(z)$ and integrate over $z$. Then again invoking the orthonormality integral, Equation (12), we arrive at the simple matrix equation

$$
\sum_{m=1}^{\infty} D_{m^{\prime} m} A_{m}=H_{m^{\prime}}
$$

Here we have defined

$$
\begin{aligned}
D_{m^{\prime} m}= & \frac{2}{L} \int_{0}^{L / 2} \mathrm{~d} z f(z) \mathcal{V}_{m^{\prime}}(\xi) \frac{\mathrm{d} \mathcal{T}_{m}(\xi)}{\mathrm{d} z} \\
H_{m^{\prime}}= & -\bar{\rho} L \sum_{m=1}^{\infty}\left[\hat{h}_{m^{\prime} m}(0)-\hat{h}_{m^{\prime} \bar{m}}(0)\right] \frac{2}{L} \\
& \times \int_{0}^{L / 2} \mathrm{~d} z f(z) \mathcal{V}_{m}(\xi) \frac{\mathrm{d} \beta v_{\mathrm{ext}}(z)}{\mathrm{d} z},
\end{aligned}
$$

and have used symmetry to simplify the integrals and the summation in Equation (28). A final matrix inversion in Equation (26) yields the coefficients $A_{m}$ for the logarithm of the density profile.

Equation (22) works out in a similar fashion, with an extra matrix multiplication at the end. Since we have $f(z) \exp \left[\beta v_{\text {ext }}(z)\right]$ as our objective, we first add and subtract $\beta v_{\text {ext }}\left(z_{2}\right)$ under the derivative $\mathrm{d} / \mathrm{d} z_{2}$ on the right-hand side of (22). This gives

$$
\begin{aligned}
\frac{\mathrm{d}}{\mathrm{d} z_{1}} \ln & {\left[f\left(z_{1}\right) \mathrm{e}^{\beta v_{\text {ext }}\left(z_{1}\right)}\right] } \\
= & \bar{\rho} \int_{-L / 2}^{L / 2} \mathrm{~d} z_{2} f\left(z_{2}\right) \hat{c}\left(0, z_{1}, z_{2}\right) \\
& \times \frac{\mathrm{d}}{\mathrm{d} z_{2}} \ln \left[f\left(z_{2}\right) \mathrm{e}^{\beta v_{\text {ext }}\left(z_{2}\right)}\right] \\
& -\bar{\rho} \int_{-L / 2}^{L / 2} \mathrm{~d} z_{2} f\left(z_{2}\right) \hat{c}\left(0, z_{1}, z_{2}\right) \frac{\mathrm{d} \beta v_{\text {ext }}\left(z_{2}\right)}{\mathrm{d} z_{2}} .
\end{aligned}
$$

Again expanding functions as in Equations (23) and (24), we now have

$$
\begin{aligned}
\sum_{m=1}^{\infty} A_{m} \frac{\mathrm{d} \mathcal{T}_{m}(\xi)}{\mathrm{d} z}= & \sum_{m=1}^{\infty} \sum_{m_{1}, m_{2}=-\infty}^{\infty} \bar{\rho} \hat{c}_{m_{1} m_{2}}(0) A_{m} \mathcal{E}_{m_{1}}(z) \\
& \times \int_{-L / 2}^{L / 2} \mathrm{~d} z^{\prime} f\left(z^{\prime}\right) \mathcal{E}_{m_{2}}^{*}\left(z^{\prime}\right) \frac{\mathrm{d} \mathcal{T}_{m}\left(\xi^{\prime}\right)}{\mathrm{d} z^{\prime}} \\
& -\sum_{m_{1}, m_{2}=-\infty}^{\infty} \bar{\rho} \hat{c}_{m_{1} m_{2}}(0) \mathcal{E}_{m_{1}}(z) \\
& \times \int_{-L / 2}^{L / 2} \mathrm{~d} z^{\prime} f\left(z^{\prime}\right) \mathcal{E}_{m_{2}}^{*}\left(z^{\prime}\right) \frac{\mathrm{d} \beta v_{\text {ext }}\left(z^{\prime}\right)}{\mathrm{d} z^{\prime}}
\end{aligned}
$$

which we once more follow with a multiplication by $(i / L) f(z) \mathcal{E}_{m^{\prime}}^{*}(z)$ and integration over $z$. This yields

$$
\begin{aligned}
& \sum_{m=1}^{\infty} \sum_{m^{\prime \prime}=-\infty}^{\infty}\left[\delta_{m^{\prime} m^{\prime \prime}}-\bar{\rho} L \hat{c}_{m^{\prime} m^{\prime \prime}(0)}\right] D_{m^{\prime \prime} m} A_{m} \\
& =-\sum_{m^{\prime \prime}=-\infty}^{\infty} \bar{\rho} \hat{c}_{m^{\prime} m^{\prime \prime}}(0) \int_{-L / 2}^{L / 2} \mathrm{~d} z f(z) \mathcal{V}_{m^{\prime \prime}}(\xi) \frac{\mathrm{d} \beta v_{\mathrm{ext}}(z)}{\mathrm{d} z}
\end{aligned}
$$

Now put

$$
\begin{aligned}
Q_{m^{\prime} m}= & \sum_{m^{\prime \prime}=-\infty}^{\infty}\left[\delta_{m^{\prime} m^{\prime \prime}}-\bar{\rho} L \hat{c}_{m^{\prime} m^{\prime \prime}}(0)\right] D_{m^{\prime \prime} m}, \\
C_{m^{\prime}}= & -\bar{\rho} L \sum_{m=1}^{\infty}\left[\hat{c}_{m^{\prime} m}(0)-\hat{c}_{m^{\prime} m}(0)\right] \\
& \times \frac{2}{L} \int_{0}^{L / 2} \mathrm{~d} z f(z) \mathcal{V}_{m}(\xi) \frac{\mathrm{d} \beta v_{\mathrm{ext}}(z)}{\mathrm{d} z},
\end{aligned}
$$

so that the $A_{m}$ are again found by a simple matrix inversion, now of

$$
\sum_{m=1}^{\infty} Q_{m^{\prime} m} A_{m}=C_{m^{\prime}}
$$

Using the OZ Equation (15), one can show that Equations (26) and (34) are identical. Calculating the $A_{m}$ separately from both could be useful as an internal numerical check.

\section{Constructing the orthogonal functions}

The familiar procedure for constructing a new set of orthogonal functions from a given complete set is the Gram-Schmidt method [15]. Thus, starting with the standard polynomials $T_{m}(\xi)=\cos [m(2 \pi z / L)]$, the first three (unnormalized) new polynomials of $\xi$ that are orthogonal with weight function $f(z)$ can be explicitly written as

$$
p_{0}(\xi)=1,
$$




$$
\begin{gathered}
p_{1}(\xi)=T_{1}(\xi)-\mu_{1} p_{0}(\xi) \\
p_{2}(\xi)=T_{2}(\xi)-\left(\frac{\mu_{3}-2 \mu_{1} \mu_{2}+\mu_{1}}{\mu_{2}-2 \mu_{1}^{2}+1}\right) p_{1}(\xi)-\mu_{2} p_{0}(\xi)
\end{gathered}
$$

where the

$$
\mu_{j}=\frac{2}{L} \int_{0}^{L / 2} \mathrm{~d} z f(z) T_{j}(\xi)
$$

are modified moments of $f(z)$. For computer calculations, however, each new application of these successive subtractions reduces the numerical precision, so that, in practice, this method becomes unusable beyond a small number of new polynomials.

Here we follow instead a robust approach described by Press and Teukolsky [21], based on works of Sack and Donovan [22] and Wheeler [23]. The monic version of the Chebyshev polynomials $T_{m}(\xi), \pi_{m}(\xi)=T_{m}(\xi) / 2^{m-1}$ for $m>0$, have by construction a leading coefficient of unity. With $\pi_{-1}(\xi) \equiv 0$ and $\pi_{0}(\xi) \equiv 1$, they satisfy a standard recursion relation,

$$
\begin{aligned}
\pi_{m+1}(\xi) & =\left(\xi-\alpha_{m}^{T}\right) \pi_{m}(\xi)-\beta_{m}^{T} \pi_{m-1}(\xi), \\
m & =0,1,2, \ldots,
\end{aligned}
$$

with $\alpha_{m}^{T}=0$ for all $m$ and $\beta_{1}^{T}=1 / 2, \beta_{m}^{T}=1 / 4$ for $m>1$. The Press-Teukolsky algorithm then uses these monic coefficients $\alpha_{m}^{T}, \beta_{m}^{T}$ along with the set

$$
v_{j}^{T}=\frac{2}{L} \int_{0}^{L / 2} \mathrm{~d} z f(z) \pi_{j}(\xi),
$$

or $v_{j}^{T}=\mu_{j} / 2^{j-1}$ for $j>0$, to recursively generate a new set of coefficients $a_{m}$ and $b_{m}$ such that, with $\mathcal{P}_{-1}(\xi) \equiv 0$ and $\mathcal{P}_{0}(\xi) \equiv 1$, the monic polynomials $\mathcal{P}_{m}(\xi)$ generated with these coefficients,

$$
\begin{aligned}
\mathcal{P}_{m+1}(\xi) & =\left(\xi-a_{m}\right) \mathcal{P}_{m}(\xi)-b_{m} \mathcal{P}_{m-1}(\xi), \\
m & =0,1,2, \ldots,
\end{aligned}
$$

are orthogonal with weight function $f(z)$. Thus to construct $n$ polynomials $\mathcal{P}_{m}(\xi)$ for $m=1$ through $m=n$, we need the coefficients $a_{m}, b_{m}$ for $m=0$ through $m=n-1$.

The key ingredient for this is the matrix [21]

$$
\sigma_{k j}=\left\langle\mathcal{P}_{k} \mid \pi_{j}\right\rangle \equiv \frac{2}{L} \int_{0}^{L / 2} \mathrm{~d} z f(z) \mathcal{P}_{k}(\xi) \pi_{j}(\xi) .
$$

(Note that $\sigma_{k j}=0$ for $k>j$.) It is clear that $\sigma_{-1, j}=0$ for all $j$ and $\sigma_{0 j}=v_{j}^{T}$ for $j=0, \ldots, 2 n-1$. Then the earlier recursions yield a recursion for the $\sigma_{k j}$,

$$
\begin{aligned}
\sigma_{k j}= & \sigma_{k-1, j+1}+\left(\alpha_{j}-a_{k-1}\right) \sigma_{k-1, j} \\
& +\beta_{j} \sigma_{k-1, j-1}-b_{k-1} \sigma_{k-2, j},
\end{aligned}
$$

for $k=1, \ldots, n-1$ and $j=k, \ldots, 2 n-k-1$. We set $a_{0}=\alpha_{0}+v_{1}^{T} / v_{0}^{T}$ and $b_{0}=0$; then putting $\sigma_{k+1, k-1}=0$ and $\sigma_{k+1, k}=0$ yields, respectively,

$$
b_{k}=\frac{\sigma_{k k}}{\sigma_{k-1, k-1}},
$$

$$
a_{k}=\alpha_{k}+\frac{\sigma_{k, k+1}}{\sigma_{k k}}-\frac{\sigma_{k-1, k}}{\sigma_{k-1, k-1}},
$$

for $k=1, \ldots, n-1$, and the $n$ monic polynomials $\mathcal{P}_{1}(\xi), \ldots, \mathcal{P}_{n}(\xi)$ are determined by recursion, Equation (41).

A final normalization yields the desired generalized Chebyshev polynomials,

$$
\begin{aligned}
\mathcal{T}_{0}(\xi) & =1, \\
\mathcal{T}_{m}(\xi) & =\frac{\mathcal{P}_{m}(\xi)}{\left[2\left\langle\mathcal{P}_{m} \mid \mathcal{P}_{m}\right\rangle\right]^{1 / 2}}, \quad m=1, \ldots, n .
\end{aligned}
$$

The algorithm further generates the roots $\xi_{1}, \xi_{2}, \ldots, \xi_{n}$ of $\mathcal{T}_{n}(\xi)=0$ and the weights $w_{j}$ for the $n$-point GaussChebyshev quadrature,

$$
\frac{2}{L} \int_{0}^{L / 2} \mathrm{~d} z f(z) F(\xi) \approx \sum_{j=1}^{n} w_{j} F\left(\xi_{j}\right)
$$

The quadrature is exact if $F(\xi)$ is a polynomial of degree less than or equal to $2 n-1$, such as in Equation (27).

A similar procedure can now be followed for the generalization $\mathcal{U}_{m}(\xi)$ of type II Chebyshev polynomials $U_{m}(\xi)$ [15]. For this we need the corresponding monic coefficients, $\alpha_{m}^{U}=0$ and $\beta_{m}^{U}=1 / 4$, for all $m$, and the modified moments

$$
v_{j}^{U}=\frac{2}{L} \int_{0}^{L / 2} \mathrm{~d} z f(z) \sin ^{2}(2 \pi z / L) \frac{U_{j}(\xi)}{2^{j}} .
$$

It is easy to show that

$$
\begin{aligned}
& v_{0}^{U}=1 / 2-v_{2}^{T}, \\
& v_{j}^{U}=v_{j}^{T} / 4-v_{j+2}^{T}, \quad j>0 .
\end{aligned}
$$

The construction of the $\mathcal{U}_{m}(\xi)$, normalized to $1 / 2$ with weight function $f(z) \sin ^{2}(2 \pi z / L)$, then follows precisely that of the $\mathcal{T}_{m}(\xi)$, so we finally get the desired $\mathcal{V}_{m}(\xi)=\sin (2 \pi z / L) \mathcal{U}_{m-1}(\xi)$.

By construction, then, these generalized Chebyshev functions $\mathcal{T}_{m}(\xi)$ and $\mathcal{V}_{m}(\xi)$ satisfy

$$
\frac{1}{L} \int_{-L / 2}^{L / 2} \mathrm{~d} z f(z) \mathcal{T}_{m}(\xi) \mathcal{T}_{m^{\prime}}(\xi)= \begin{cases}1, & m=m^{\prime}=0, \\ 1 / 2, & m=m^{\prime} \neq 0, \\ 0, & m \neq m^{\prime},\end{cases}
$$


$\frac{1}{L} \int_{-L / 2}^{L / 2} \mathrm{~d} z f(z) \mathcal{V}_{m}(\xi) \mathcal{V}_{m^{\prime}}(\xi)= \begin{cases}0, & m=m^{\prime}=0 \\ 1 / 2, & m=m^{\prime} \neq 0 \\ 0, & m \neq m^{\prime}\end{cases}$

$\frac{1}{L} \int_{-L / 2}^{L / 2} \mathrm{~d} z f(z) \mathcal{T}_{m}(\xi) \mathcal{V}_{m^{\prime}}(\xi)=0, \quad$ for all $m, m^{\prime}$

Collectively, Equations (50)-(52) give rise to the orthonormalization of $\mathcal{E}_{m}(z)$ expressed in Equation (12).

\section{An illustration}

In this section, we illustrate some practical details regarding the formal tailored polynomials discussed above. As noted earlier, actual applications will be left to later work.

For purposes of illustration we choose a system of hard spheres of diameter $\sigma$ between parallel hard walls a distance $L$ apart $[6,9]$. The external potential is then

$$
\beta v_{\mathrm{ext}}(z)= \begin{cases}0, & |z| \leq(L-\sigma) / 2 \\ \infty, & (L-\sigma) / 2<|z| \leq L / 2\end{cases}
$$

so that the initial (non-interacting) density profile of the system is

$$
f(z)=\frac{L}{L-\sigma} H\left(\frac{L-\sigma}{2}+z\right) H\left(\frac{L-\sigma}{2}-z\right),
$$

where $H(x)$ is the Heaviside unit step function and the prefactor is chosen for normalization, Equation (13). In this case, we can calculate the needed $v_{j}^{T}=$ $(2 / L) \int_{0}^{L / 2} \mathrm{~d} z f(z) \pi_{j}(\xi)$ analytically. We obtain

$$
\begin{gathered}
v_{0}^{T}=1, \\
v_{j}^{T}=\frac{L \sin (j \pi \sigma / L)}{j \pi(L-\sigma)}\left(-\frac{1}{2}\right)^{j-1}, \quad j>0,
\end{gathered}
$$

and the appropriate $\mathcal{T}_{m}[\cos (2 \pi z / L)]$ are then constructed following the recipe in Section 4.

We have arbitrarily chosen $L=5 \sigma$ for these calculations and show in Figure 1 the first four special polynomials $\mathcal{T}_{1}(\xi), \ldots, \mathcal{T}_{4}(\xi)$ generated for this case (omitting the trivial $\mathcal{T}_{0}(\xi)=1$ ). They are seen to be quite changed from the original constant-amplitude Chebyshev form, $T_{m}(\xi)=\cos [m(2 \pi z / L)]$. Similarly, the corresponding first four generalizations of $V_{m}(\xi)=\sin [m(2 \pi z / L)], \quad \mathcal{V}_{1}(\xi), \ldots, \mathcal{V}_{4}(\xi), \quad$ seen $\quad$ in Figure 2, also display variable amplitudes, though of course they still vanish at the end points. Note that the integral common to both Equation (28) and (33) picks

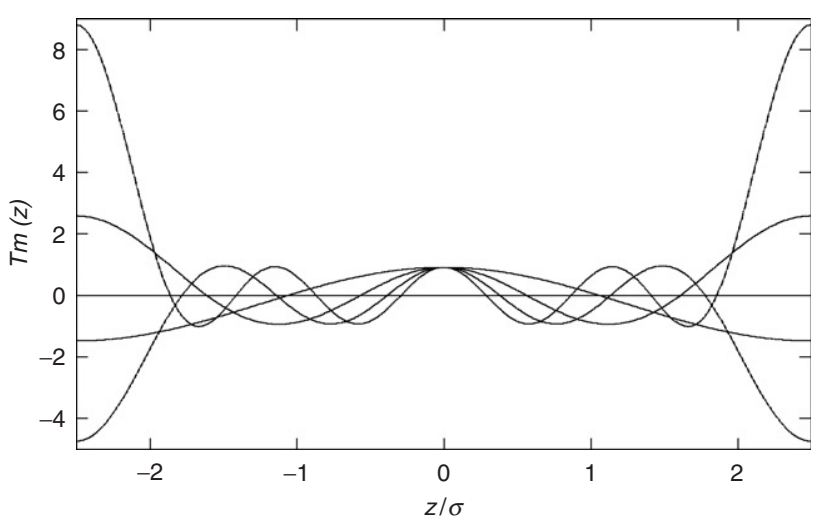

Figure 1. Tailored orthogonal polynomials $\mathcal{T}_{1}(\xi), \mathcal{T}_{2}(\xi)$, $\mathcal{T}_{3}(\xi), \mathcal{T}_{4}(\xi)$ generalizing the Chebyshev $T_{m}(\xi)=\cos [m(2 \pi z)$ $L)$ ] constructed for the rectangular (ideal) density profile of Equation (54) for slit width $L=5 \sigma$; here $\xi \equiv \cos (2 \pi z / L)$.

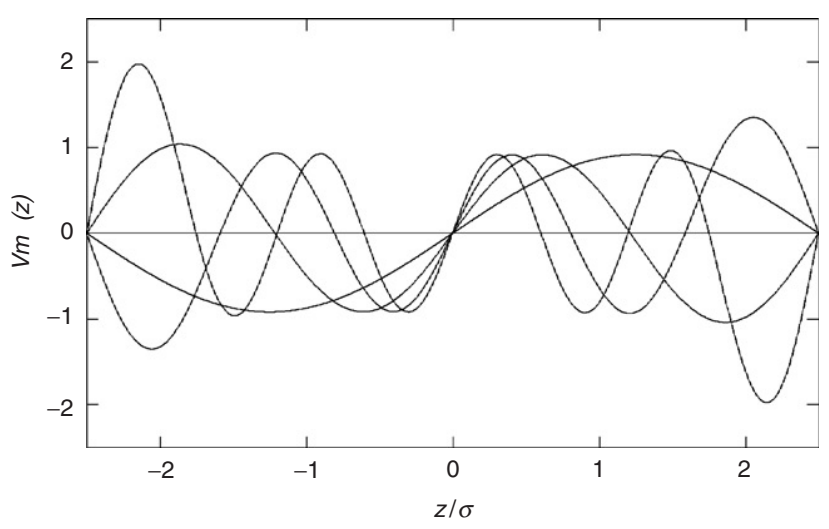

Figure 2. Tailored orthogonal functions $\mathcal{V}_{1}(\xi), \mathcal{V}_{2}(\xi), \mathcal{V}_{3}(\xi)$, $\mathcal{V}_{4}(\xi)$ generalizing the Chebyshev $V_{m}(\xi)=\sin [m(2 \pi z / L)]$ constructed for the rectangular (ideal) density profile of Equation (54) for slit width $L=5 \sigma$; here $\xi \equiv \cos (2 \pi z / L)$.

up the values of these latter functions at the cutoff, $z=(L-\sigma) / 2$ :

$$
\begin{aligned}
\frac{2}{L} & \int_{0}^{L / 2} \mathrm{~d} z f(z) \mathcal{V}_{m}(\xi) \frac{\mathrm{d} \beta v_{\text {ext }}(z)}{\mathrm{d} z} \\
& =-\frac{2}{L} \int_{0}^{L / 2} \mathrm{~d} z f(z) \mathrm{e}^{\beta v_{\text {ext }}(z)} \mathcal{V}_{m}(\xi) \frac{\mathrm{de}^{-\beta v_{\text {ext }}(z)}}{\mathrm{d} z} \\
& =\frac{2}{L} \int_{0}^{L / 2} \mathrm{~d} z f(z) \mathrm{e}^{\beta v_{\text {ext }}(z)} \mathcal{V}_{m}(\xi) \delta\left(z-\frac{L-\sigma}{2}\right) \\
& =\frac{2}{L-\sigma} \mathcal{V}_{m}\left[\cos \left(\frac{L-\sigma}{L} \pi\right)\right]
\end{aligned}
$$

It is clear from the earlier sections that the procedure proposed here at least yields a satisfying simplification of the formalism used to solve the prescribed equations for a full description of an inhomogeneous fluid. The practical question, however, is whether it 
also produces a significant reduction in computing time for these solutions, perhaps even putting such calculations on a par with those of homogeneous systems. This is essentially a question of how the number of coefficients, say $n$, used in an expansion like (23) compares with the number of grid points, say $N_{z}$, needed for an equally good representation of the same function directly on the $z$ axis. In a real calculation, Equation (23) would be used to find $\ln \left\{f(z) \exp \left[\beta v_{\text {ext }}(z)\right]\right\}$ from the calculated $A_{m}$. Here we are going to turn that around, create a realistic model of a continuous density profile on the $z$ grid, and see how many coefficients $A_{m}$ are required to reproduce it.

For the modeled density profile we turn to the Percus-Yevick (PY) equation for hard spheres [20]. Specifically, we start with the continuous function $g_{\mathrm{HS}}(r) \exp \left[\beta v_{\mathrm{HS}}(r)\right]$ that the PY equation produces at $\rho \sigma^{3}=0.8$ with a grid interval $\Delta r=0.02 \sigma$, cut out the section from $r=0.5 \sigma$ to $r=3.0 \sigma$, and call that the left half of our modeled density profile, $f_{0}(z) \exp \left[\beta v_{\mathrm{ext}}(z)\right]$, over the interval $z=-2.5 \sigma$ to $z=2.5 \sigma$ with $\Delta z=0.02 \sigma$. Then we simply fold that piece over to produce the symmetric right half; the result is shown in Figure 3. This continuous density profile is not a very familiar quantity. If, however, we now truncate this shape at $\pm(L-\sigma) / 2$ (that is, multiply by $\exp \left[-\beta v_{\text {ext }}(z)\right]$ ), we get the discontinuous density profile $f_{0}(z)$ seen in Figure 4. This is indeed a familiar quantity. Specifically, it strongly resembles (by design) the density profile computed by Kjellander and Sarman [9] for a hard sphere fluid between hard walls at separation $L=5 \sigma$ and shown as their Figure 1(e). Thus, the data for Figure 3 will serve as our realistic test profile. It is based on $N_{z}=250$ grid points; we then use fourthorder interpolation to obtain the test density profile at the $n$ points needed for the Gauss-Chebyshev quadrature below.

Applying the expansion of (23) to our test profile, we have

$$
\ln \left[f_{0}(z) \mathrm{e}^{\beta v_{\text {ext }}(z)}\right]=\sum_{m=0}^{n-1} A_{m} \mathcal{T}_{m}(\xi)
$$

where we are still using the special polynomials $\mathcal{T}_{m}(\xi)$ constructed for the $f(z)$ of Equation (54) and sampled in Figure 1. Thus, multiplying this equation by $(1 / L)$ $f(z) \mathcal{T}_{k}(\xi)$ and integrating over $z$ produces, for $k>0$,

$$
\begin{aligned}
A_{k} & =\frac{2}{L} \int_{-L / 2}^{L / 2} \mathrm{~d} z f(z) \ln \left[f_{0}(z) \mathrm{e}^{\beta v_{\text {ext }}(z)}\right] \mathcal{T}_{k}(\xi) \\
& =2 \sum_{j=1}^{n} w_{j} \ln \left[f_{0}\left(z_{j}\right) \mathrm{e}^{\beta v_{\text {ext }}\left(z_{j}\right)}\right] \mathcal{T}_{k}\left(\xi_{j}\right),
\end{aligned}
$$

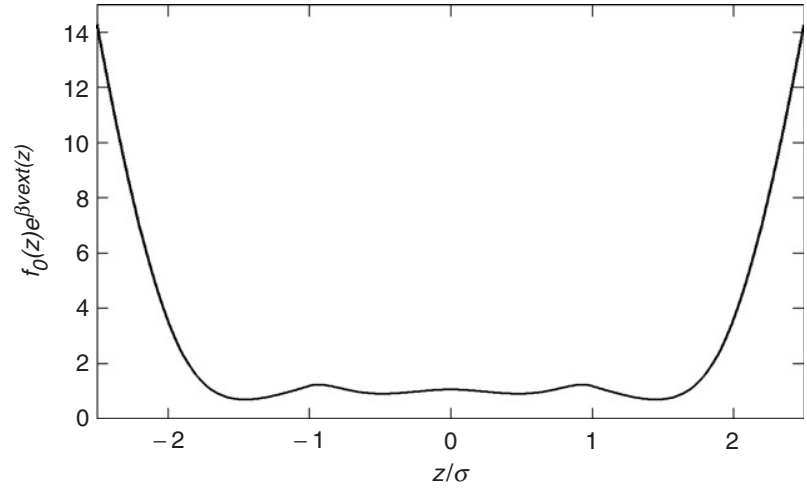

Figure 3. Continuous test profile $f_{0}(z) \exp \left[\beta v_{\text {ext }}(z)\right]$ for hard spheres between hard walls with a slit width $L=5 \sigma$, constructed using $g_{\mathrm{HS}}(r) \exp \left[\beta v_{\mathrm{HS}}(r)\right]$ from the PercusYevick equation for hard spheres at $\rho \sigma^{3}=0.8$. See text.

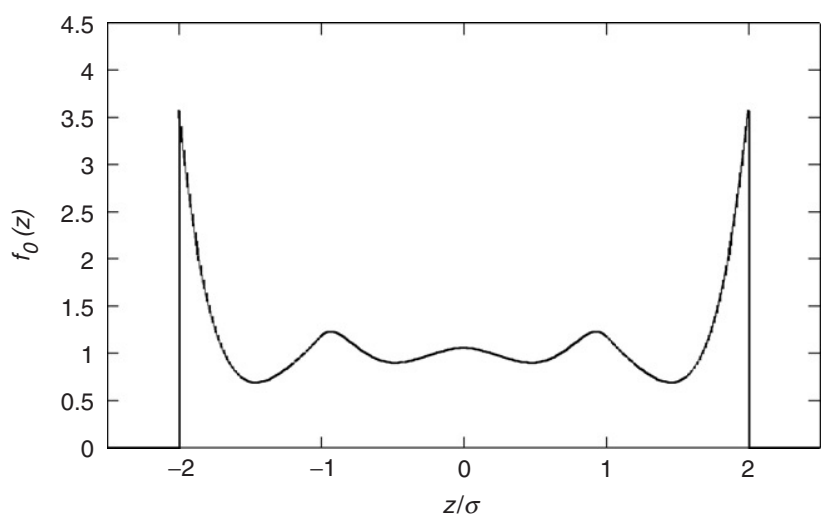

Figure 4. Discontinuous test profile $f_{0}(z)$ for hard spheres between hard walls with a slit width $L=5 \sigma$ obtained by truncation of the form in Figure 3 at $z= \pm(L-\sigma) / 2$.

after invoking Equations (50) and (47). The quadrature is now evaluated for successive values of $k$; the results are shown graphically as $A_{m}$ vs. $m$ in Figure 5, for which we have used $n=30$. It seems clear that, holding $n$ fixed at 30 , fewer than the 30 coefficients $A_{m}$ (numbered 0 through 29) seen in the figure would actually suffice in the expansion (58). In fact, we anticipate that the cutoff limits for single index expansions such as (23) or double index expansions such as (14) may be set at some integer $n_{c}$ that is meaningfully smaller than the given $n$, which is kept larger to set the parameters for the Gauss-Chebyshev quadratures.

We conclude that the number $n_{c}$ of coefficients needed in the tailored orthogonal functions procedure put forward in this work will be about an order of magnitude smaller than the number of grid points $N_{z}$ needed for the direct space procedures used in earlier works. Since much of the numerical effort involves 


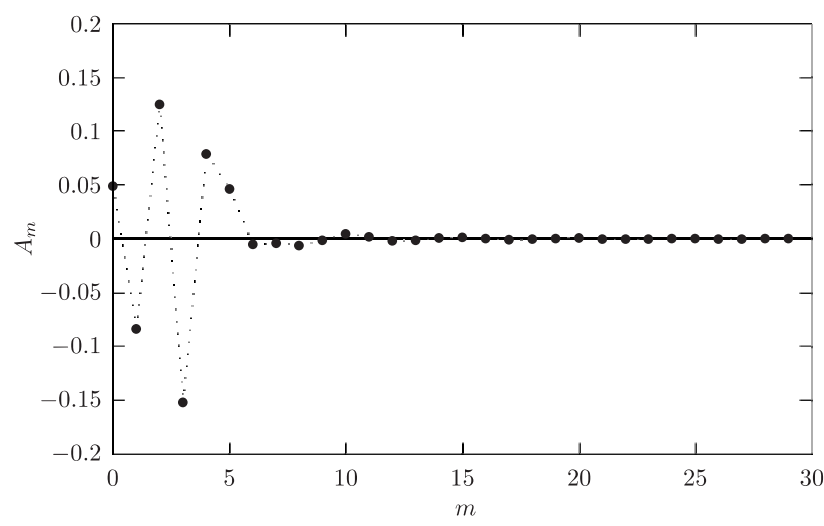

Figure 5. Coefficients $A_{m}$ for the expansion of Equation (58) as a function of integer $m$.

matrix operations, of order $N_{z}^{2}$ in Equation (6) vs. $n_{\mathrm{c}}^{2}$ in Equation (15), we may reasonably conclude that solutions obtained using the tailored orthogonal functions will require on the order of $1 \%$ of the computational effort of those earlier works. That should make equations for inhomogeneous fluids as readily solvable as their more familiar homogeneous cousins.

\section{References}

[1] D. Henderson, in Fundamentals of Inhomogeneous Fluids, edited by D. Henderson (Dekker, New York, 1992), Chap. 4.

[2] S. Sokołowski, J. Chem. Phys. 73, 3507 (1980).

[3] S. Sokołowski, Mol. Phys. 49, 1481 (1983).

[4] M. Plischke and D. Henderson, Proc. R. Soc. Lond. A 404, 323 (1986).
[5] M. Plischke and D. Henderson, J. Chem. Phys. 84, 2846 (1986).

[6] R. Kjellander and S. Sarman, Chem. Phys. Lett. 149, 102 (1988).

[7] R. Kjellander and S. Sarman, Mol. Phys. 70, 215 (1990).

[8] R. Kjellander and S. Sarman, Mol. Phys. 74, 665 (1991).

[9] R. Kjellander and S. Sarman, J. Chem. Soc. Faraday Trans. 87, 1869 (1991).

[10] P. Attard, J. Chem. Phys. 91, 3072 (1989).

[11] P. Attard, J. Chem. Phys. 91, 3083 (1989).

[12] S.A. Egorov, J. Chem. Phys. 112, 7138 (2000).

[13] E. Lomba, S. Jorge, and M. Álvarez, Phys. Rev. E 63, 011203 (2000).

[14] J.M. Brader, J. Chem. Phys. 128, 104503 (2008).

[15] G. Arfken, Mathematical Methods for Physicists (Academic Press, Orlando, 1985).

[16] M.S. Green, J. Chem. Phys. 33, 1403 (1960); ibid. 39, 1367 (1963). Green classified the diagrams in the density expansion of $g(r) \mathrm{e}^{\beta v(r)}$ by analogy with electric circuits as 'series', 'parallel', or 'bridge', the last because of the resemblance of the first diagram to a Wheatstone bridge. The 'parallel' terms can be summed in direct space and disappear. The name 'series' is nowadays seldom used but the 'bridge' name incongruously survives.

[17] D.G. Triezenberg and R. Zwanzig, Phys. Rev. Lett. 28, 1183 (1972).

[18] R. Lovett, C.Y. Mou, and F.P. Buff, J. Chem. Phys. 65, 570 (1976).

[19] M.S. Wertheim, J. Chem. Phys. 65, 2377 (1976).

[20] J.P. Hansen and I.R. McDonald, Theory of Simple Liquids (Academic Press, London, 1986).

[21] W.H. Press and S.A. Teukolsky, Comp. Phys. 4, 423 (1990).

[22] R.A. Sack and A.F. Donovan, Num. Math. 18, 465 (1971/72).

[23] J.C. Wheeler, Rocky Mount. J. Math. 4, 287 (1974). 